\title{
Impact of Mouse Model on Preclinical Dosimetry in Targeted Radionuclide Therapy
}

Samir Boutaleb ${ }^{12}$, Jean-Pierre Pouget ${ }^{13^{*}}$, Cecilia Hindorf ${ }^{4}$, André Pèlegrin ${ }^{1}$, Jacques Barbet ${ }^{2}$, Pierre-Olivier Kotzki ${ }^{1}$, Manuel Bardiès ${ }^{24}$

1 IRCM, Institut de recherche en cancérologie de Montpellier INSERM : U896, Université Montpellier I, CRLCC Val d'Aurelle - Paul Lamarque , F-34298 Montpellier,FR

2 CRCNA, Centre de Recherche en Cancérologie Nantes - Angers INSERM : U892, Université de Nantes, CHU Nantes, FR

3 IRSN, Institut de radioprotection et de sûreté nucléaire Ministère de l'écologie de l'Energie, du Développement durable et de l'Amé nagement du territoire, Ministère de l'économie, de l'industrie et de l'emploi, Ministère de l'Enseignement Supérieur et de la Recherche Scientifique, Ministère de la Défense, Ministère de la santé , BP1792262 Fontenay-aux-Roses Cedex,FR

${ }^{4}$ Ecole Nationale Vétérinaire de Nantes Ministère de l'Agriculture et de la Pêche, ONIRIS Site de la Chantrerie BP 4070644307 Nantes cedex $3, F R$

* Correspondence should be adressed to: Jean-Pierre Pouget <jean-pierre.pouget@ valdorel.fnclcc.fr >

\section{Abstract \\ Introduction}

Small animal dosimetry serves as an important link in establishing a relationship between absorbed dose and biological effect during pre-clinical targeted radionuclide therapy. Dosimetric approaches reported to date are based on models aiming at representing the animals used during pre-clinical experiments. However, anatomical variations between models may generate differences in the dosimetric results. Our goal was to assess the impact of the mouse model on the absorbed dose per cumulated activity (S value, in Gy per Bq.s).

Methodology

Two datasets were considered. The first one was developed in our laboratory and is a voxel-based model of a $30 \mathrm{~g}$ female nude mouse. Images were segmented manually to identify more than 30 organs and sub-organs. The second dataset originates from the DIGIMOUSE project. In that model, a $28 \mathrm{~g}$ normal male nude mouse was used to generate the segmented structures of 9 regions in the head and 12 major organs.

A software developed in our laboratory allowed us to read each 3D mouse atlas slice by slice, to crop the mouse volume to remove background air voxels, and to write the geometry description as an input file for the Monte-Carlo code MCNPX using "repeated structure representation". A linear interpolation scales the voxel size as a function of the total body mass.

Results

The comparison of various voxel-based mouse dosimetric models shows that even when scaled to the same total-body mass, models from different mouse breed or gender demonstrate very different organ masses, volumes, and therefore $\mathrm{S}$ values.

Conclusions

Computation of the $\mathbf{S}$ values for pre-clinical studies depends strongly on the definition of the mouse model. Our computational model is a step in the direction of a more realistic description of the geometry in pre-clinical dosimetry.

Author Keywords Dosimetry ; Monte-Carlo simulation ; Small Animal ; Targeted Radionuclide- Therapy (TRT) ; Voxel-Based Models

\section{INTRODUCTION}

Targeted radionuclide therapy (TRT) is a cancer treatment modality in which a biologic vector conveys a radioactive isotope to the neighborhood of cancer targets, thus concentrating radiations with the ultimate goal of selectively irradiating tumor cells [1 ]. The vector can be the nuclide itself, as is the case in the treatment of differentiated thyroid cancer with 131-iodine. The vector can be a monoclonal antibody targeting antigens specific to cancer cells (thus defining radioimmunotherapy), a metabolite or a peptide. The radionuclide must preserve the targeting specificity by emitting short range radiations. Beta emitters are usually used for this purpose, even though the potential of alpha or Auger emitters is being considered [2 ]. Targeted radionuclide therapy has demonstrated its potential in the therapy of radiosensitive non-Hodgkins B-lymphoma [3 ]-[4 ], melanoma [5 ] or neuroendocrine tumors [6 ].

Small animal dosimetry serves as an important link in establishing a relationship between absorbed dose and biological effect (toxicity or efficacy) during pre-clinical targeted radionuclide therapy. 
The absorbed dose can be derived according to the MIRD formalism [7 ]:

$$
\bar{D}_{(k-h)}=\tilde{A}_{h} \cdot S_{(k-h)}
$$

- where $\mathrm{D}_{(\mathrm{k} \leftarrow \mathrm{h})}$ is the mean absorbed dose in gray $(\mathrm{Gy})$ to the target $\mathrm{k}$ from radiation emitted by the source $\mathrm{h}$,

- $\AA_{\mathrm{h}}$ is the cumulated activity in becquerel-seconds (Bq.s) in the source region $\mathrm{h}$ and

- $\mathrm{S}_{(\mathrm{k} \leftarrow \mathrm{h})}$ is the mean absorbed dose in gray per becquerel-second (Gy/Bq.s) to the target $\mathrm{k}$ per unit cumulated activity in the source $\mathrm{h}$ (or $\mathrm{S}$ value).

As can be seen from the above equation, absorbed dose calculation requires both the cumulated activity and the $\mathrm{S}$ value to be determined.

The cumulated activity is usually determined from measurements of activity in organs/tissues at different time points after injection of a radiopharmaceutical. Animals are sacrificed and dissected and the organ/tissue is weighed and measured for activity thus yielding the mean/standard deviation of activity concentration at various time points. The selected animals are usually of the same age and strain for better homogeneity of the results. The cumulated activity is calculated as the time integral of the activity as a function of time after the injection.

Computational phantoms based on a realistic anatomy are needed to quantify organ average absorbed doses [8 ]. Several authors have proposed murine S values, using stylized phantoms (mathematical models) [9 ]-[12] and more recently anatomical voxel-based models [13 ]-[15 ]. This is an important step since, contrary to the situation in clinical dosimetry, the range of the $\beta$ particles involved in TRT is of the same order of magnitude as the dimensions of the small animal organs and tissues of interest. S values should therefore not be calculated under the assumption that $\beta$ radiation is non-penetrating (the absorbed fraction equals 1). An explicit model of radiation transport has to be considered for most organ/tissues of interest in murine dosimetry. In addition, even in situations where the activity distribution is homogeneous within source organs, the absorbed dose distribution is likely to be heterogeneous because of the finite range of the beta particle, thus warranting a voxel-based absorbed dose calculation.

Since cumulated activities are obtained by averaging measurements obtained from several animals, it is usual to consider $\mathrm{S}$ values obtained from a reference mouse or rat model, rather than on the specific animal that has been used for the experiment. Still, several arguments questioning the relevance of the murine model have been raised including:

- Different strains of mice yield animals of different sizes and shapes. Organ geometries might be affected, thus producing different $\mathrm{S}$ values. It is therefore important to use $S$ values that have been developed for the animal strain considered in the study, or to be able to adjust $\mathrm{S}$ values computed for a given animal model to a more relevant one.

- Tumor models cannot be explicitly integrated within a "reference" mouse model. Tumor dosimetry must therefore be considered separately.

More recently, quantitative imaging has paved the way for longitudinal studies [16 ], allowing the pharmacokinetics to be determined for each individual animal. The accuracy of such an approach remains to be ascertained. Operational issues such as the ability to sequentially anesthetize the same animal safely, or to fuse images obtained at different time points with accuracy on par with the spatial resolution of the imaging devices have to be addressed [17 ]. The radiation burden resulting from the sequential use of microCT is generally not lethal, but the absorbed dose levels may be sufficient to induce deterministic effects that could confound the biological outcome of the experiment [18 ]. Still, it must be considered that animal-specific pharmacokinetics are or will be available within the near future from micro imaging devices (micro-PET/CT or micro-SPECT/CT). In that context, the relevance of model-based dosimetry may have to be reconsidered: If cumulated activities can be obtained for a given animal, then on principle the absorbed dose should be determined for the animal itself rather than for a model, unless it can be proven that using a model does not impact the dosimetric results markedly.

Absorbed dose calculations can be performed using different approaches. Monte-Carlo codes can be used to follow radiation transport and score energy deposition. When compared to less refined approaches such as Dose Point Kernel convolutions, Monte-Carlo approaches have the advantage of being able to correctly account for the heterogeneous materials (soft tissue, bone, lung, air) present within animal bodies. The price to pay (independently of the inherent complexity of most Monte-Carlo packages available to the scientific community) is that Monte-Carlo-based calculations usually require intensive computing capabilities, thus restricting the adoption of the approach to a limited number of laboratories. 
The main goal of this work was therefore to assess the impact of anatomical variation on murine $\mathrm{S}$ values. Two murine dosimetric models developed in our laboratory were considered. Our models were scaled to other models presented in the literature, so that $\mathrm{S}$ values could be compared for different mouse models of the same total body-weight.

\section{MATERIALS AND METHODS}

\section{Model definition}

This work exploited two 3D segmented datasets created according to the following protocol: A mouse was embedded into a frozen block which was then mounted on the moving table of a cryo-microtome and sectioned into $100 \mu \mathrm{m}$ slices. High-resolution digital photographs were taken of each section. The first dataset was developed by Bitar et al. in our laboratory [13 ]. It is based on a $30 \mathrm{~g}$ Naval Medical Research Institute (NMRI) female athymic nude mouse. Manual image segmentation led to the identification of 30 regions (organs and sub-organs). Original data was stored in the tiff format, with each pixel index number representing an organ or sub-organ (bladder wall, bladder content, left and right limb bones, brain, colon, heart, left and right kidney medulla, left and right kidneys cortex, liver, left and right lungs, bone marrow in femurs and legs, left and right ovaries, pancreas, skull, small intestine, spleen, spinal cord, stomach wall, stomach contents, left and right suprarenal glands, thyroid, uterus, vertebrae, fat, and carcass). Additionally, tumors have to be inserted into this geometry to represent particular pre-clinical conditions [19].

The second dataset originates from the Digimouse project [20 ] and is based on a $28 \mathrm{~g}$ normal nude male mouse. The 3D atlas, initially designed for brain imaging, is composed of 9 brain regions and 12 other organs (whole brain, external cerebrum, cerebellum, olfactory bulbs, striatum, medulla, massetter muscles, eyes, lachrymal glands, heart, lungs, liver, stomach, spleen, pancreas, adrenal glands, kidneys, testes, bladder, skeleton and skin). The complete dataset is available on http://neuroimage.usc.edu/Digimouse.html .

Fig. 1 presents an axial and a sagital view of the two murine models considered in this study: The Digimouse male-model (Fig. 1A ) and the Bitar et al. female-model (Fig. 1B ).

In this study, our results were compared with data published previously by Stabin et al. [14 ] and Larsson et al. [15 ]. The model considered by Stabin et al. is a transgenic mouse (body mass $27 \mathrm{~g}$ ), imaged using a dedicated small-animal CT scanner $(256 \times 256 \times 256$ matrix with voxel size $0.2 \mathrm{~mm}$ ). Identified organs were segmented from the CT slices (kidneys, liver, lungs, spleen, heart, stomach, intestines, skeleton, testes, and bladder). The second study used the Moby phantom [21 ], based on a segmented C57BL/6 mouse (body mass 33g). 3D magnetic resonance microscopy (MRM) was used to create non-uniform rational b-spline (NURBS) representing the organ shapes. A matrix of $128 \times 128 \times 432$ elements with a voxel size of $0.25 \mathrm{~mm}$ was generated. Organ masses for that anatomical atlas were calculated by Taschereau et al. [22 ] in order to derive absorbed doses for use within the context of 18-fluorine compounds used in PET imaging experiments.

Our two mouse datasets were down-sampled to match the scale of the other models [14 ], [15 ]. The female phantom was stored in a matrix of $220 \times 450 \times 111$ voxels of $0.222 \times 0.222 \times 0.2 \mathrm{~mm}^{3}$. The nude male atlas was stored using $190 \times 496 \times 104$ matrix elements, with a voxel size of $0.2 \mathrm{~mm}$. Tissue densities and chemical composition were taken from ICRU report 44 [23 ], except for the bladder wall, bladder content, colon, small intestine, stomach wall and stomach contents, which were obtained from Woodard et al. [24 ]. Table 1 summarizes the characteristics of the models considered in this study and Table 2 provides the density references used for each model.

\section{MCNPX Monte-Carlo simulations}

The previous work published by Bitar et al. was performed using the MCNP4c2 Monte-Carlo code [26 ]. However, more recent versions of MCNP and MCNPX 2.5 [27 ] have implemented new features, such as scoring and source sampling using a repeated structures representation that increases computing performance for voxel-based geometries. In order to check the consistency of the results produced by the two versions of the code, we first computed $S$ values with the two codes while keeping the geometrical model and isotope data constant.

We calculated S values from absorbed energy distributions obtained with the Monte-Carlo N-Particle MCNPX transport code. All simulations were run on a MacBookPro (Apple) $2.16 \mathrm{GHz}$ Intel Core 2 Duo with 3 GB RAM. A variance reduction technique was used and all particles transported from a region of higher importance (organ regions) to a region of lower importance (background air voxels) underwent a geometry splitting with Russian roulette [26 ]. Preliminary tests insured that implementing variance reduction did not modify the end results. Each mouse organ was simulated as a radiation source, assuming a uniform activity distribution in each. Radionuclide energy spectra were taken from ICRP 38 [28 ]. The number of histories was chosen so as to keep the statistical uncertainties below $3 \%$.

Variations between different mouse models 
The models considered in this study are based on different mouse strains, but have similar masses, viz.: $30 \mathrm{~g}$ for the female athymic nude mouse, $28 \mathrm{~g}$ for the normal nude male mouse, $27 \mathrm{~g}$ for the model proposed by Stabin et al. and $33 \mathrm{~g}$ for MOBY (Table 1 ). In order to reduce the influence of the global volume of each model studied, we decided to scale the various models to the same total mass.

For that purpose, an interactive editing tool was developed specifically: "model generator". The software's main goals are the fast and automatic generation of an input file for the Monte-Carlo code (MCNP or MCNPX) used to simulate radiation transport and score energy deposition. The tool reads the 3D atlas slice by slice and crops around the mouse volume to remove background air voxels. A label associates each voxel to a defined tissue type, with a density value and a material description. The whole body mass is entered into the model generator. Voxel dimensions are then scaled linearly to create a new dataset corresponding to the total body mass required. The model generator allows indexing and counting of organ voxels. Organ masses are obtained by multiplying the number of voxels associated with the tissue type by tissue density and voxel volume. The model generator ultimately creates the MCNPX input files from the atlas data.

To make a comparison with the results of studies by Stabin et al. and Larsson et al. the absorbed fractions for 100-keV photons originating from the liver, and $1-\mathrm{MeV}$ electrons originating from the lungs, were calculated for our 2 models (the male nude mouse from Digimouse and the female nude mouse from Bitar et al. ) :

- Male-model and female-model scaled to 27g (Table 3 ) and compared to the results presented by Stabin et al.

- Male-model and female-model scaled to 33g, and compared to the results presented by Larsson et al (Table 4 ).

- In order to compare the results obtained from our 2 models, ${ }^{131} \mathrm{I} \mathrm{S}$ values were calculated for the female-model scaled to $30 \mathrm{~g}$ and compared to the results presented by Bitar et al. (Table 5 ). Additionally, $\mathrm{S}$ values for ${ }^{131} \mathrm{I}$ were generated from the original Digimouse model $(28 \mathrm{~g})$ and compared to the results obtained from the same male-model scaled at $30 \mathrm{~g}$ (Table 6 ).

All comparisons were made on a subset of organs identified in all models: liver, skeleton, stomach, kidneys, lungs, heart, spleen, pancreas, bladder, testes (for males [14 ], [15 ], [20 ]), ovaries (for female [13 ]) and the remainder of the body.

\section{RESULTS}

\section{Comparison of S values from MCNP4c2 Versus MCNPX}

Our approach using the model generator was compared to previously published result, with the same voxel-based model [19] but different MCNP code versions. As an example, Table 7 shows the S values of three source/target organ combinations (kidneys, lungs and liver) for 131 -iodine. The differences in the $\mathrm{S}$ values (Gy/Bq.s) were less than $\pm 2 \%$. Additionally, the gain in terms of computing speed was greater than $50 \%$ as compared to original MCNP4c2 runtimes. Since both simulations were run on the same hardware/operating system, this computing efficiency increase can be attributed to the implementation of variance reduction techniques and algorithmic improvements introduced in recent MCNP versions [29].

\section{Impact of mouse weight}

Fig. 2 and 3 present the absorbed fractions (AFs) obtained by Monte-Carlo modeling for $100 \mathrm{keV}$ photon sources located in the liver for the dosimetric mouse models considered in the study:

Our two models (female-model proposed by Bitar et al. and male-model from Digimouse) were scaled to $27 \mathrm{~g}$ using our model generator in order to match the total weight of the mouse model proposed by Stabin et al. , and then scaled to $33 \mathrm{~g}$ in order to match the total weight of the mouse model proposed by Larsson et al. As can be seen from tables 2 and 3 , the scaling process did not entirely compensate for the differences in organ weights observed with different mouse strains or gender. This translates into varying AFs, even for models of an equivalent total mass. The self-absorbed fractions (liver to liver) were significantly different $\left(1.47 \mathrm{E}^{-2}, 1.24 \mathrm{E}^{-2}\right.$ and $\left.9.32 \mathrm{E}^{-3}\right)$ for the $27 \mathrm{~g}$ models (Fig 1 ) and closer $\left(1.58 \mathrm{E}^{-2}, 1.33 \mathrm{E}^{-2}\right.$ and $1.7 \mathrm{E}^{-2}$ ) for the $33 \mathrm{~g}$ models (Fig 2 ), and generally followed liver mass variations. Cross-absorbed fractions also varied markedly between models, except for some combinations where target organ masses were close (liver to kidneys for Bitar's and Stabin's models in Fig. 2 , liver to heart for Digimouse and Bitar's model in Fig. 2 and 3 ).

Fig. 4 and 5 represent the absorbed fractions obtained by Monte-Carlo simulation for $1 \mathrm{MeV}$ electron sources located in the lungs for the dosimetric mouse models considered in this study. Values lower than $1 \mathrm{E}^{-4}$ were set to zero on the graph. Self-absorbed fractions were usually similar between models, a fact that can be explained by the smaller range of electrons in tissues. Cross-absorbed fractions varied between models (for example in the case of lung to heart, or lung to skeleton), a fact that can be explained by the different methods used to segment datasets. Additionally, it must be stressed that the ribs and sternum (i.e. skeletal bones near the lungs) were not segmented in the model of Bitar et al. . 
Table 5 shows the ratios of the $\mathrm{S}$ values generated by two models of the same strain (nude mouse) with the same whole body mass, post-scaling. The Digimouse voxel-based model was scaled using the model generator from $28 \mathrm{~g}$ to $30 \mathrm{~g}$ in order to match the female-model total body mass. The differences in organ masses before scaling were important $(0.134 \mathrm{~g}$ vs. $0.123 \mathrm{~g}$ for lungs, $2.64 \mathrm{~g}$ vs. $1.83 \mathrm{~g}$ for liver and $0.652 \mathrm{~g}$ vs. $0.754 \mathrm{~g}$ for kidneys), and the transformation from a $28 \mathrm{~g}$ to a $30 \mathrm{~g}$ model did not compensate for the original differences. The resulting differences in terms of S values were very pronounced (between 7.47 and $43.13 \%$ ). Indeed, organ size and shapes do differ between the two models, as can be seen from the visualization of the phantoms in Fig. 1(A and B).

Table 6 compares 131-Iodine $\mathrm{S}$ values from the original male mouse model ( $28 \mathrm{~g}$ ) against $\mathrm{S}$ values for the same model scaled to $30 \mathrm{~g}$. The total-body mass scaled model demonstrates different organ masses, volumes, and shapes.

\section{DISCUSSION}

The model-based mouse dosimetry literature has been recently extended by the presentation of three voxel-based models representing different mouse strains (Stabin et al. [14 ], Bitar et al. [13 ] and Larsson et al. [15 ]). In this work, we also introduced a male nude mouse dosimetric model based on the Digimouse atlas [20].

Since it has been shown previously that varying mouse sizes may lead to important $S$ values variations [12 ] we developed a mouse model generator that scales a given model to a predetermined total mass, and generates a MCNPX input file in order to reduce computation times by eliminating segmentation stage. However, it appears that the linear total-body mass scaling does not correctly model the variations in organ size, shape and location. Consequently absorbed fractions or S values obtained from the various scaled models can be very different.

In addition to differences in strain or gender, the production of the voxel representation of the various models was not equivalent: Stabin's model was obtained from microCT slices of a living animal, segmented with standard image processing tools available on a commercial workstation. No reference to the original animal strain was given in the article. The transgenic nature of the mouse considered in Stabin's study may explain the unusually low weight of the liver $(0.78 \mathrm{~g})$. MOBY's original dataset was obtained from images of a living animal (using MRI), whereas our two dosimetric models were obtained by manual segmentation of cryosections of a dead animal. Imaging and segmentation methodologies, combined with differences in original strains and gender probably all contribute to the observed variations in organ shape and mass, even for total-body mass scaled models. Interestingly, even very similar models (Digimouse and Bitar' s model) with similar original total-body weights (28g and 30g) still demonstrate quite different organ masses.

This raises several questions regarding the relevance of "reference models" for mouse dosimetry.

If the purpose of a given study is to compare the dosimetry of different isotopes (for example high vs. low energy beta emitters), then the use of a similar geometry - hence a reference mouse model - to perform the comparison is probably wise.

In all other situations, the dosimetric model should match as closely as possible the geometric characteristics of the mouse strain of interest for the study. A linear transformation such as that conducted in our study may not be sufficient to "adapt" a single model to different mouse strains.

Some further issues should be investigated. First, animals selected for a given pre-clinical experiment are usually chosen from amongst mice of the same age and strain. The study of the variation in organ masses and shape in a similar animal batch is underway in order to determine if a "representative" mouse from the experimental batch may be used as a reference for dosimetric studies. This might lead to a range of "representative" mouse models, a possibility that can be considered due to current computation capabilities and the finite number of animal strains used in pre-clinical experiments. Another area to explore is the possibility of adapting our model generator to give a correct interpolation between mice from the same strain but differing in age, or even between mice from different strains. This would require implementing more advanced registration methods than linear interpolation. As an alternative, adapting NURBS-based phantoms, like MOBY for example, is certainly worth consideration.

Mouse-specific dosimetry is yet another possibility to explore. In that situation, mouse specific biokinetics obtained from sequential quantitative imaging would be used to derive absorbed dose estimates for every animal under investigation. As was seen in the introduction of this article, real mouse-specific dosimetry still represents a scientific challenge, for many reasons. However, as far as the absorbed dose calculation alone is concerned, the use of state of the art Monte-Carlo codes such as those used in our study, that couple sound and reliable radiation transport physics with dedicated algorithms to increase computation speed in a voxel-based geometry is becoming a viable option. Computation times required to perform whole-body absorbed dose calculations are now of the order of hours using commercially available computers, a value that can easily be reduced by the use of computer clusters that are remarkably efficient for "embarrassingly parallel problems" such as those involved in absorbed dose calculations. 


\section{CONCLUSION}

In a previous publication, we introduced a voxel-based dosimetric model of a female nude mouse [13 ]. In the current article, we present a nude male mouse dosimetric model. A software tool was developed to facilitate the creation of additional murine dosimetric models based on our two original datasets by scaling the total-body mass. However, comparison between our models and those presented by other authors demonstrates that even total-body mass scaled models exhibit different organ masses, volumes, and shapes. This obviously leads to variations in calculated S values. Additional work is in progress to determine if, within the same strain and for animals of the same age, geometric variations between samples for the same experimental batch are small enough to warrant the generation of a representative mouse dosimetric model, for a given type of pre-clinical experiment. A possible alternative would be to use image-based mouse geometry and pharmacokinetics to derive mouse-specific absorbed dose calculations. This certainly is feasible from a computing perspective. Still, mouse-specific dosimetry needs to be validated as a viable option for pre-clinical experiments.

\section{Ackowledgements:}

The authors thank Dr. E McKay for checking the manuscript.

This work was supported by the "Comité de l'Hérault de la Ligue Nationale de Lutte Contre le Cancer, Montpellier, France ".

\section{References:}

- 1. Chatal JF, Hoefnagel CA . Radionuclide therapy . Lancet . 354 : Sep 1999; 931 - 935

- 2 . Pouget J , Santoro L, Raymond L, Chouin N, Bardiès M, Bascoul-Mollevi C, Huguet H, Azria D, Kotzki P, Pèlegrin M , Vivès E, Pèlegrin A . Cell Membrane is a More Sensitive Target than Cytoplasm to Dense Ionization Produced by Auger Electrons . Radiat Res . 170 : Aug 2008 ; 192 - 200

- 3 . Capizzi RL . Targeted radio-immunotherapy with Bexxar produces durable remissions in patients with late stage low grade non-Hodgkin's lymphomas . Trans Am Clin Climatol Assoc . $115: 2004 ; 255-72$

- 4. Macklis RM . Radioimmunotherapy as a therapeutic option for Non-Hodgkin's lymphoma . Semin Radiat Oncol . 17 : Jul $2007 ; 176$ - 83

- 5 . Miao Y, Quinn TP . Peptide-targeted radionuclide therapy for melanoma . Crit Rev Oncol Hematol . 67 : Sep 2008 ; 213 - 28

- 6. Lewington VJ . Targeted radionuclide therapy for neuroendocrine tumours . Endocr Relat Cancer . 10 : Dec $2003 ; 497$ - 501

- 7 . Loevinger R, Budinger TF, Watson EE . Mird Primer for Absorbed Dose Calculations .

- 8. Zaidi H , Xu XG . Computational anthropomorphic models of the human anatomy: the path to realistic Monte Carlo modeling in radiological sciences . Annual Review of Biomedical Engineering . 9:2007; $471-500$

- 9. Hui TE, Fisher DR, Kuhn JA, Williams LE, Nourigat C, Badger CC, Beatty BG, Beatty JD . A mouse model for calculating cross-organ beta doses from yttrium-90-labeled immunoconjugates . Cancer. 73 : Feb $1994 ; 951$ - 7

- 10. Flynn AA, Green AJ , Pedley RB , Boxer GM , Boden R, Begent RH . A mouse model for calculating the absorbed beta-particle dose from (131)I- and (90)Y-labeled immunoconjugates, including a method for dealing with heterogeneity in kidney and tumor. Radiat Res . 156: Jul 2001;28 - 35

- 11. Kolbert KS, Watson T, Matei C, Xu S, Koutcher JA, Sgouros G . Murine S factors for liver, spleen, and kidney . J Nucl Med . 44 : May 2003 ; 784 - 91

- 12. Hindorf C, Ljungberg M, Strand S . Evaluation of parameters influencing S values in mouse dosimetry . J Nucl Med . 45 : Nov 2004 ; 1960 - 5

- 13. Bitar A, Lisbona A, Thedrez P, Sai Maurel C, Le Forestier D, Barbet J, Bardies M . A voxel-based mouse for internal dose calculations using Monte Carlo simulations (MCNP). Phys Med Biol . 52 : Feb $2007 ; 1013$ - 25

- 14. Stabin MG, Peterson TE, Holburn GE, Emmons MA . Voxel-based mouse and rat models for internal dose calculations . J Nucl Med . 47 : Apr 2006 ; 655 - 9

- 15. Larsson E, Strand S, Ljungberg M, Jönsson B. Mouse S-factors based on Monte Carlo simulations in the anatomical realistic Moby phantom for internal dosimetry . Cancer Biother Radiopharm . 22 : Jun $2007 ; 438$ - 42

- 16. Tornai MP, Jaszczak RJ, Turkington TG, Coleman RE . Small-animal PET: advent of a new era of PET research . J Nucl Med . 40 : Jul 1999 ; 1176 - 1179

- 17 . Hildebrandt IJ , Su H, Weber WA. Anesthesia and other considerations for in vivo imaging of small animals . ILAR J . 49 : 2008 ; 17 - 26

- 18 . Carlson SK, Classic KL, Bender CE, Russell SJ . Small animal absorbed radiation dose from serial micro-computed tomography imaging . Molecular Imaging and Biology: MIB: The Official Publication of the Academy of Molecular Imaging . 9 : $2007 ; 78-82$

- 19. Bitar A, Lisbona A, Bardiès M. S-factor calculations for mouse models using Monte-Carlo simulations . Q J Nucl Med Mol Imaging . 51 : Dec 2007 ; 343 - 51

- 20 . Dogdas B , Stout D, Chatziioannou AF, Leahy RM . Digimouse: a 3D whole body mouse atlas from CT and cryosection data . Physics in Medicine and Biology . 52 : $2007 ; 577-587$

- 21. Segars WP, Tsui BMW, Frey EC, Johnson GA, Berr SS . Development of a 4-D digital mouse phantom for molecular imaging research . Mol Imaging Biol . 6 : Jun $2004 ; 149-59$

- 22. Taschereau R, Chatziioannou AF. Monte Carlo simulations of absorbed dose in a mouse phantom from 18-fluorine compounds . Med Phys . 34 : Mar 2007 ; 1026 - 1036

- 23 . ICRU Report 44: Tissue Substitutes in Radiation Dosimetry and Measurement . Bethesda, MD 1989;

- 24. Woodard HQ, White DR. The composition of body tissues . Br J Radiol . 59 : Dec 1986; 1209 - 18

- 25 . Zubal IG, Harrell CR, Smith EO, Rattner Z, Gindi G, Hoffer PB . Computerized three-dimensional segmented human anatomy . Med Phys . 21 : Feb 1994 ; 299 - 302

- 26 . Briesmeister JF . MCNP - A General Monte Carlo N-Particle Transport Code --- Version 4C . Los Alamos, NM Los Alamos National Laboratory ; Report LA-13709-M 2000 ;

- 27 . Hendricks JS, McKinney GW, Waters LS, Roberts TL, Egdorf HW, Finch JP, Trellue HR, Pitcher EJ, Mayo DR, Swinhoe MT . MCNPX extensions version 2.5. 0 . Los Alamos, NM Los Alamos National Laboratory ; Report LA-UR-04-0570 2004 ;

- 28. Radionuclide transformations. Energy and intensity of emissions. Report of a Task Group of Committee 2 of the International Commission on Radiological Protection on data used in ICRP Publication 30. Ann ICRP . 11-13: 1983; 1 - 1250

- 29. Chiavassa S, Bardies M, Franck D , Jourdain J , Chatal J . Computer Time (CPU) comparison of several input file formats considering different versions of MCNPX in case of personalised voxel-based Dosimetry . presented at the proceedings of the Advanced Mathematical and Computational Tools in Metrology and testing (AMCTM) Caparica, Portugal Jun. 2005 


\section{Figure 1}

Axial and sagital views of (A) Digimouse scaled at $30 \mathrm{~g}$, and (B) original (30 g) Bitar et al. model (B) using MCNP viewer tool.
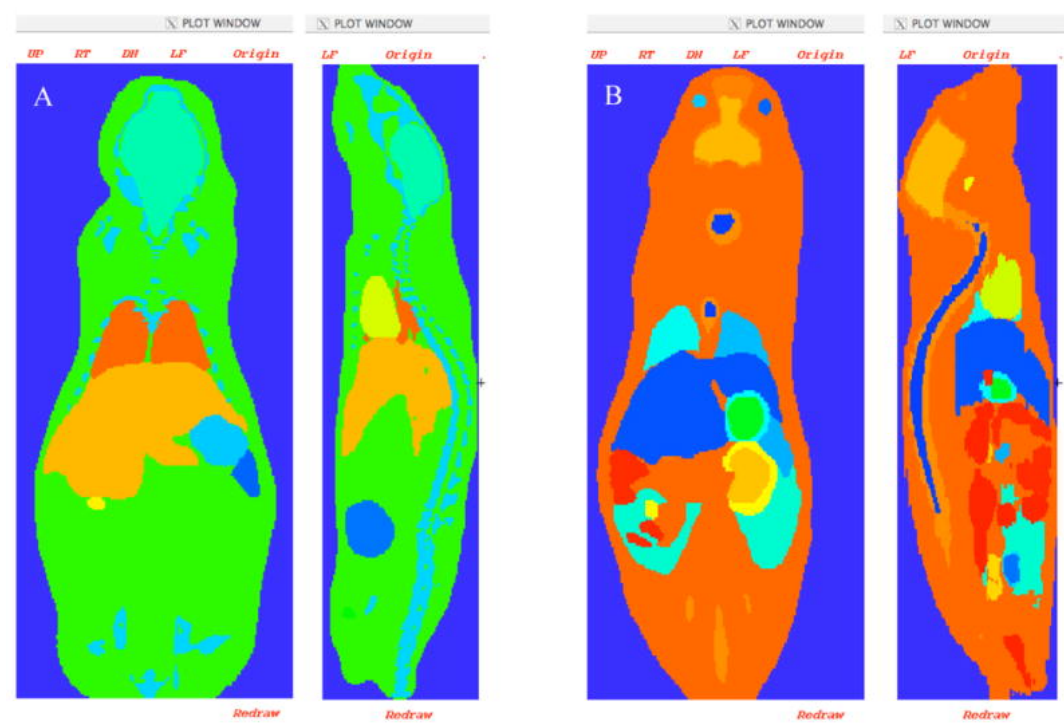

\section{Figure 2}

Comparison of absorbed fractions from 100-keV photons originating from the liver, as calculated by the dosimetry model of Stabin et al. and models of this work: male mouse (Digimouse) and female scaled to 27-g.

\section{$100 \mathrm{kev}$ photons in Liver}
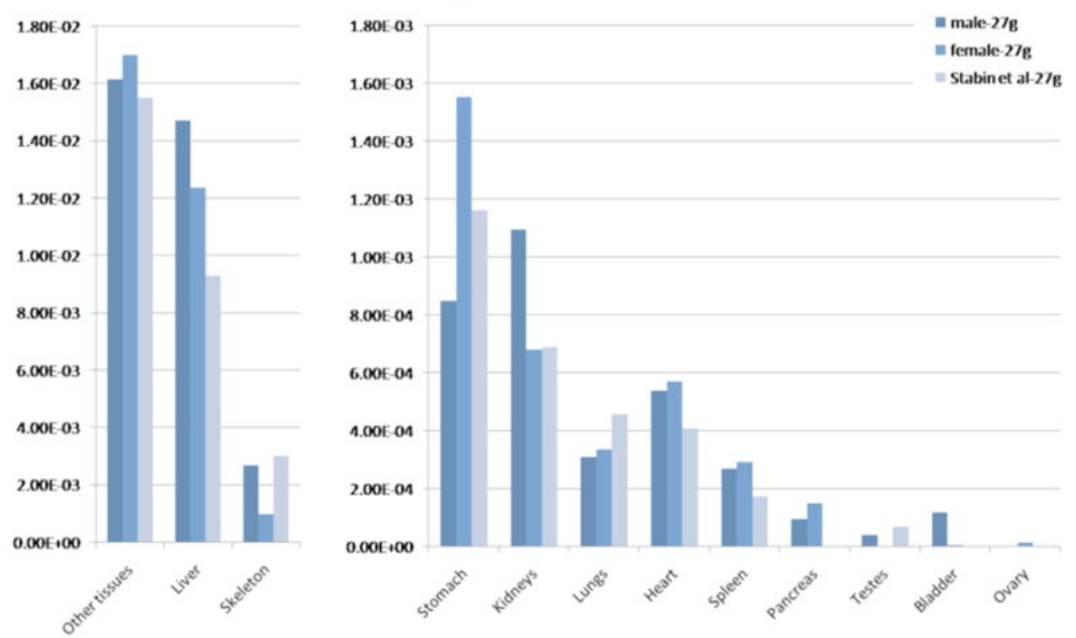

\section{Figure 3}

Comparison of absorbed fractions from 100-keV photons originating from the liver, as calculated by the dosimetry model of Larsson et al. (Moby) and models of this work: male mouse (Digimouse) and female scaled to 33-g.

\section{$100 \mathrm{kev}$ photons in Liver}
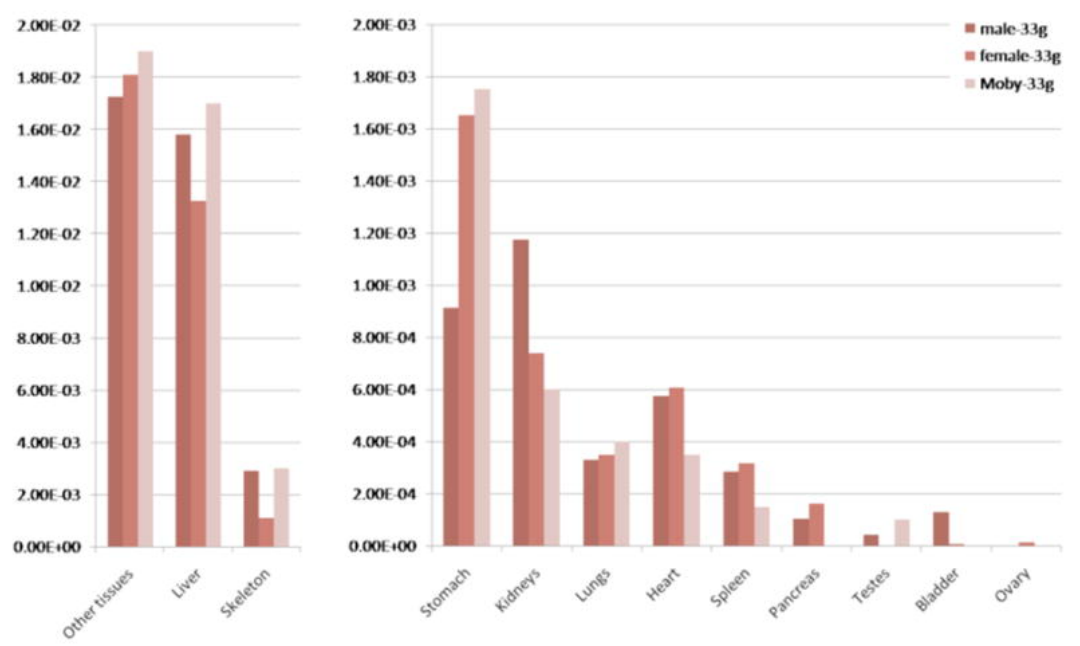
Figure 4

Comparison of absorbed fractions from 1-MeV electrons originating from the lung, as calculated by the dosimetry model of Stabin et al. and models of this work: male mouse (Digimouse) and female scaled to 27-g.

\section{$1 \mathrm{MeV}$ electrons in Lung}

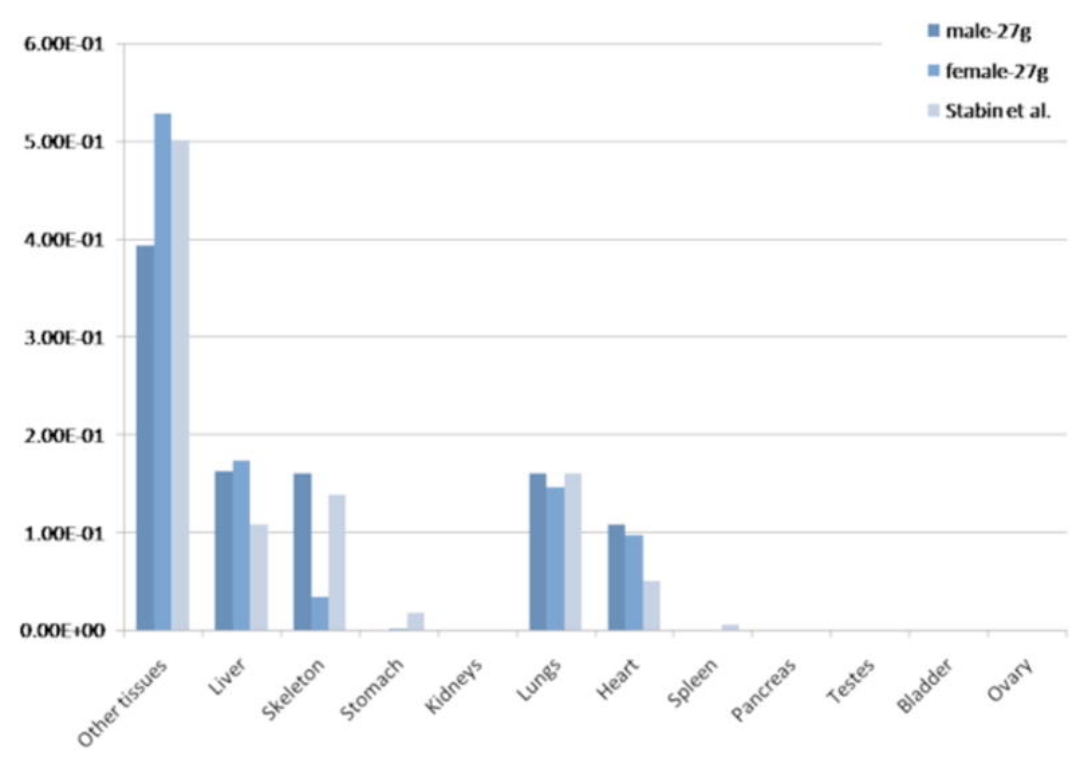

\section{Figure 5}

Comparison of absorbed fractions from 1-MeV electrons originating from the lung, as calculated by the dosimetry model of Larsson et al. (Moby) and models of this work: male mouse (Digimouse) and female scaled to 33-g.

\section{$1 \mathrm{MeV}$ electrons in Lung}

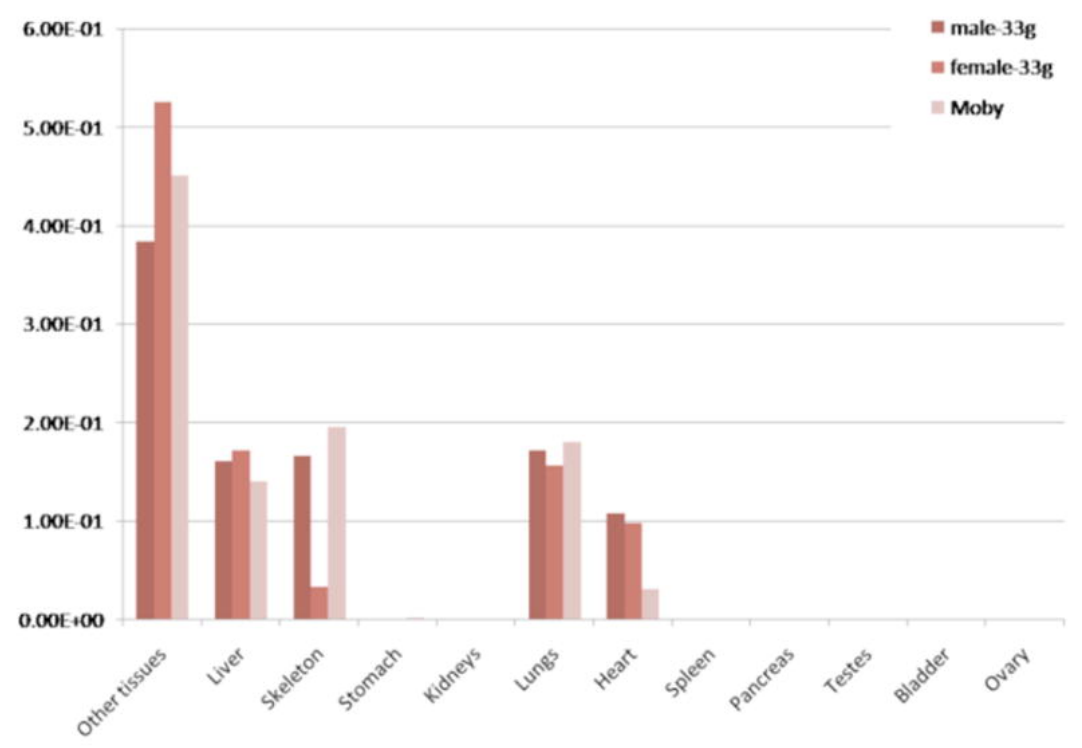


Table 1

Characteristics of the models considered in this study.

\begin{tabular}{|c|c|c|c|c|}
\hline Reference & Bitar et al. $\left[{ }^{13}\right]$ & Digimouse $\left[{ }^{20}\right]$ & Stabin et al. [ ${ }^{14}$ ] & Larsson et al. [ ${ }^{15}$ ] \\
\hline Mouse strain & Female Nude & Male nude & Transgenic & C57BL/6 \\
\hline Mouse weight & $30-\mathrm{g}$ & $28-\mathrm{g}$ & $27-g$ & $33-g$ \\
\hline Matrix size & $220 \times 450 \times 111$ & $190 \times 496 \times 104$ & $256 \times 256 \times 256$ & $128 \times 128 \times 432$ \\
\hline Voxel size (mm) & $0.222 \times 0.222 \times 0.2$ & $0.2 \times 0.2 \times 0.2$ & $0.2 \times 0.2 \times 0.2$ & $0.25 \times 0.25 \times 0.25$ \\
\hline
\end{tabular}

Table 2

Density of mouse tissue $\left(\mathrm{g} / \mathrm{cm}^{3}\right)$

\begin{tabular}{|c|c|c|c|}
\hline \multirow[b]{2}{*}{ Organs } & \multicolumn{3}{|c|}{ Density tissue $\left(\mathrm{g} / \mathrm{cm}^{3}\right)$ of models } \\
\hline & MOBY $\S$ & Male/female * & Stabin et al. ${ }^{\Delta}$ \\
\hline$\overline{\text { Body }}$ & 1 & 1.04 & 1.04 \\
\hline Adipose (fat) & 0.92 & 0.95 & 1.04 \\
\hline Lung & 0.3 & 0.26 & 0.296 \\
\hline Heart & 1.05 & 1.06 & 1.04 \\
\hline Kidney & 1.05 & 1.05 & 1.04 \\
\hline Liver & 1.06 & 1.06 & 1.04 \\
\hline Spleen & 1.06 & 1.06 & 1.04 \\
\hline Intestine & 1.03 & 1.03 & 1.04 \\
\hline Brain & 1.04 & 1.04 & 1.04 \\
\hline Skull & 1.61 & 1.85 & 1.4 \\
\hline Spine Bone & 1.42 & 1.85 & 1.4 \\
\hline Rib Bone & 1.92 & 1.85 & 1.4 \\
\hline \multicolumn{4}{|c|}{$\begin{array}{l}\S \text { Data from log Moby; } \\
{ }^{*} \text { Data from ICRU44; } \\
{ }^{\Delta} \text { Data from Zubal et al. }\left[{ }^{25} \text { ]. }\right.\end{array}$} \\
\hline \multicolumn{4}{|c|}{ 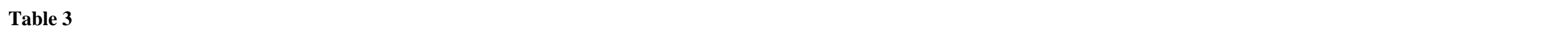 } \\
\hline \multirow[b]{2}{*}{ Organ } & \multicolumn{3}{|c|}{ Organ mass (g) for Phantoms at 27-g } \\
\hline & Scaled male (Nude normal) & Scaled female (Nude NMRI) & Stabin et al. $\left[{ }^{14}\right]$ (Transgenic) \\
\hline$\overline{\text { Liver }}$ & 2.373 & 1.596 & 0.780 \\
\hline Stomach & 0.264 & 0.382 & 0.298 \\
\hline Kidneys & 0.586 & 0.338 & 0.334 \\
\hline Lungs & 0.121 & 0.107 & 0.125 \\
\hline Heart & 0.264 & 0.246 & 0.143 \\
\hline Spleen & 0.164 & 0.189 & 0.022 \\
\hline Testes & 0.176 & I & 0.141 \\
\hline
\end{tabular}


Table 4

Scaled masses of the male and female mouse models considered in the study. The total body masses are set to $33 \mathrm{~g}$, i.e. that of the model used by Larsson et al. [ ${ }^{15}$ ] (Moby).

\begin{tabular}{|c|c|c|c|}
\hline \multirow[b]{2}{*}{ Organ } & \multicolumn{3}{|c|}{ Organ mass (g) for Phantom at 33g } \\
\hline & Scaled male (Nude normal) & Scaled female (Nude NMRI) & Moby $\left[{ }^{13}\right]($ C57BL/6) \\
\hline Liver & 2.901 & 1.951 & 2.69 \\
\hline Stomach & 0.323 & 0.466 & I \\
\hline Kidneys & 0.717 & 0.413 & 0.415 \\
\hline Lungs & 0.148 & 0.131 & 0.13 \\
\hline Heart & 0.323 & 0.300 & 0.12 \\
\hline Spleen & 0.201 & 0.231 & 0.13 \\
\hline Testes & 0.215 & / & 0.4 \\
\hline
\end{tabular}

Table 5

$\mathrm{S}$ values (Gy/Bq.s) for ${ }^{131} \mathrm{I}$, generated for the original model proposed by Bitar et al. and for Digimouse scaled to $30 \mathrm{~g}$.

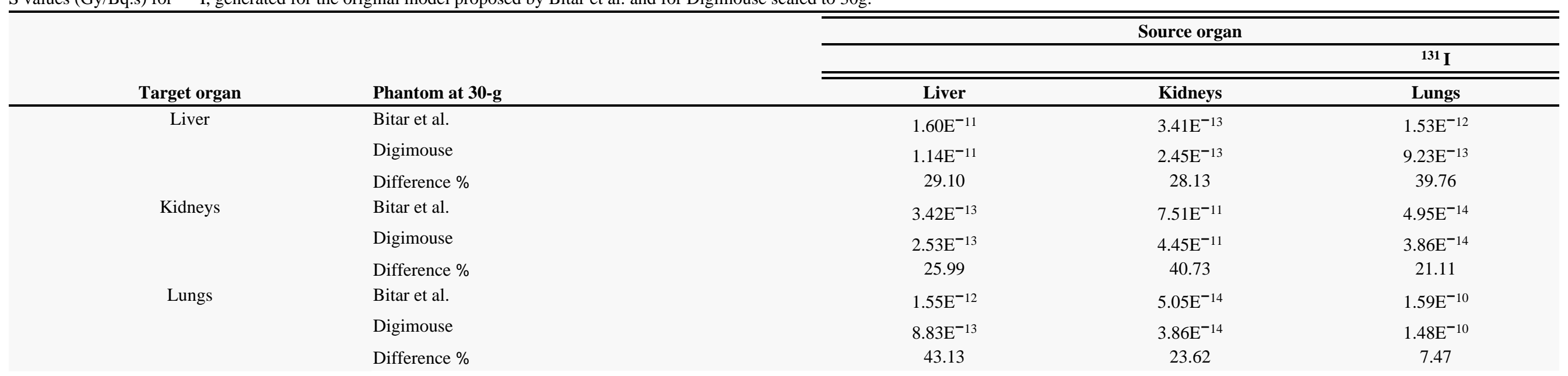


Table 6

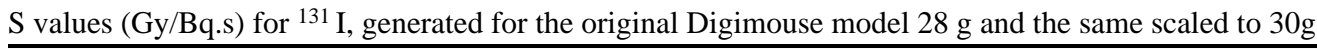

\begin{tabular}{|c|c|c|c|c|}
\hline \multirow[b]{3}{*}{ Target organ } & \multirow[b]{3}{*}{ Phantom Digimouse } & \multicolumn{3}{|c|}{ Source organ } \\
\hline & & & & ${ }^{131} I$ \\
\hline & & Liver & Kidneys & Lungs \\
\hline \multirow[t]{3}{*}{ Liver } & $28 \mathrm{~g}$ & $1.21 \mathrm{E}^{-11}$ & $2.64 \mathrm{E}^{-13}$ & $9.96 \mathrm{E}^{-13}$ \\
\hline & Scale at $30 \mathrm{~g}$ & $1.14 \mathrm{E}^{-11}$ & $2.45 \mathrm{E}^{-13}$ & $9.23 \mathrm{E}^{-13}$ \\
\hline & Difference \% & 6.43 & 7.24 & 7.34 \\
\hline \multirow[t]{3}{*}{ Kidneys } & $28 \mathrm{~g}$ & $2.60 \mathrm{E}^{-13}$ & $4.76 \mathrm{E}^{-11}$ & $3.98 \mathrm{E}^{-14}$ \\
\hline & Scale at $30 \mathrm{~g}$ & $2.53 \mathrm{E}^{-13}$ & $4.45 \mathrm{E}^{-11}$ & $3.86 \mathrm{E}^{-14}$ \\
\hline & Difference \% & 2.47 & 6.45 & 3.13 \\
\hline \multirow[t]{3}{*}{ Lungs } & $28 \mathrm{~g}$ & $1.01 \mathrm{E}^{-12}$ & $4.01 \mathrm{E}^{-14}$ & $1.57 \mathrm{E}^{-10}$ \\
\hline & Scale at $30 \mathrm{~g}$ & $8.83 \mathrm{E}^{-13}$ & $3.86 \mathrm{E}^{-14}$ & $1.48 \mathrm{E}^{-10}$ \\
\hline & Difference \% & 12.21 & 3.66 & 5.86 \\
\hline
\end{tabular}

Table 7

Comparison of ${ }^{131}$ I S values (Gy/Bq.s) obtained by MCNP4c2 and MCNPX 2.5 for the Nude NMRI model.

\begin{tabular}{|c|c|c|c|c|}
\hline \multirow[b]{3}{*}{ Target organ } & \multirow[b]{3}{*}{ MCNP version } & \multicolumn{3}{|c|}{ Source organ } \\
\hline & & \multicolumn{3}{|r|}{${ }^{131} I$} \\
\hline & & Liver & Kidneys & Lungs \\
\hline \multirow[t]{3}{*}{ Liver } & MCNP4c2 & $1.60 \mathrm{E}^{-11}$ & $3.40 \mathrm{E}^{-13}$ & $1.56 \mathrm{E}^{-12}$ \\
\hline & MCNPX & $1.60 \mathrm{E}^{-11}$ & $3.41 \mathrm{E}^{-13}$ & $1.53 \mathrm{E}^{-12}$ \\
\hline & Difference \% & -0.18 & -0.07 & 1.77 \\
\hline \multirow[t]{2}{*}{ Kidney } & $\mathrm{MCNP} 4 \mathrm{c} 2$ & $3.41 \mathrm{E}^{-13}$ & $7.48 \mathrm{E}^{-11}$ & $4.93 \mathrm{E}^{-14}$ \\
\hline & Difference \% & -0.34 & -0.43 & -0.50 \\
\hline \multirow[t]{3}{*}{ Lungs } & MCNP4c2 & $1.58 \mathrm{E}^{-12}$ & $4.96 \mathrm{E}^{-14}$ & $1.58 \mathrm{E}^{-10}$ \\
\hline & MCNPX & $1.55 \mathrm{E}^{-12}$ & $5.05 \mathrm{E}^{-14}$ & $1.59 \mathrm{E}^{-10}$ \\
\hline & Difference \% & 1.73 & -1.91 & -0.93 \\
\hline
\end{tabular}

